\title{
Femoral fracture in pregnancy: a case series and review of clinical management
}

This article was published in the following Dove Press journal:

International Journal of Women's Health

\section{Justin A Harold \\ Erin Isaacson \\ Anna Palatnik}

Department of Obstetrics and Gynecology, Medical College of Wisconsin, Milwaukee, WI, USA
Correspondence: Anna Palatnik Department of Obstetrics and Gynecology, Medical College of

Wisconsin, 9200 West Wisconsin

Avenue, Milwaukee, WI 53226, USA

Tel + I 4148056600

Fax + I 4I48056622

Email apalatnik@mcw.edu

\begin{abstract}
Femoral fractures in pregnancy are a rare complication, with an incidence of approximately $1 \%$. Case reports and small trials leave perioperative obstetric management and route of delivery largely unclear. Three cases of femoral fracture are presented that occurred at 24,30 , and 31 weeks of gestation. The causes of femoral fracture were gunshot, motor vehicle collision, and fragility fracture. All fractures were surgically repaired, 1 utilizing neuraxial anesthesia and the others with general anesthesia. A 30-day postoperative course of low-molecular-weight heparin was prescribed, and all patients subsequently had vaginal deliveries. Femoral fractures in the viable pregnancy confer high maternal and fetal morbidity and mortality. Intraoperative fetal monitoring and postoperative anticoagulation should be considered, and vaginal delivery should not be contraindicated.
\end{abstract}

Keywords: femoral fracture, antepartum testing, trauma in pregnancy, venous thromboembolism prevention, osteoporosis in pregnancy

\section{Introduction}

Orthopedic injury in pregnancy, though rare, is associated with significant morbidity and mortality to the mother and fetus. ${ }^{1}$ Some reports estimate that the risk of intrauterine fetal demise (IUFD) is as high as $40.1 \%$, depending on the location of the fracture. Pelvic and acetabular fractures convey the highest morbidity and mortality. $^{2-4}$ We present three cases of femoral fracture that occurred at our institution over a period of 2 years and discuss teaching points of perioperative obstetric management, postoperative venous thromboembolism (VTE) prophylaxis, and route of delivery. Local institutional review board approval was not required for this case series. Written consent was obtained from each patient to publish the case details.

\section{Cases}

Patient 1: A 31-year-old gravida 5 para 4 woman at 24 weeks and 4 days of gestation presented to the emergency department after having suffered 5 gunshot wounds to her bilateral lower extremities. On admission, her Glasgow Coma Scale Score was 15 and she was hemodynamically stable. Plain film radiography of the pelvis and left femur was performed. There was a comminuted, segmented, displaced fracture of the left mid-femoral diaphysis with multiple osseous and metallic fragments as well as a nearby dominant bullet fragment (Figure 1A). There were no additional injuries. Her obstetric history consisted of four previous pregnancies with uncomplicated term spontaneous vaginal deliveries. The patient reported no 

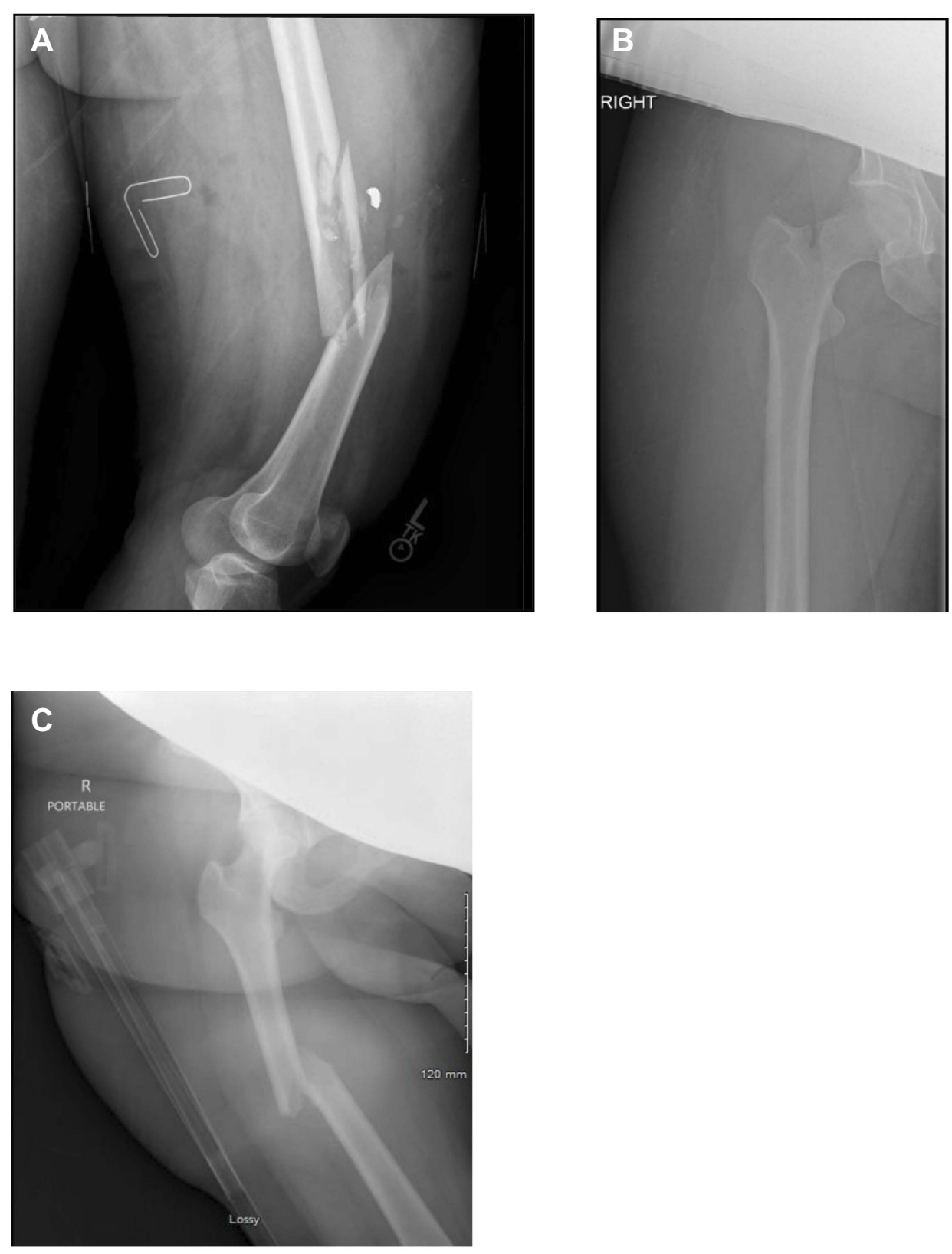

Figure I (A) Comminuted, segmented, displaced fracture with nearby dominant bullet fragment. (B) Basicervical femoral neck fracture. (C) Mildly comminuted and displaced femoral shaft fracture with foreshortening.

additional medical problems or prior surgical history. Laboratory evaluation was significant for a low serum calcium level of $8.2 \mathrm{mg} / \mathrm{dL}$.

The patient underwent retrograde intramedullary nailing under general endotracheal anesthesia. Intraoperative fetal monitoring demonstrated moderate variability with occasional shallow variable decelerations. The total radiation dosage both preoperatively and intraoperatively was 20.1 milligray (mGy). A lead apron was used to shield the maternal abdomen during all images required pre- andintraoperatively. In the postoperative care unit, uterine tocometry showed contractions every 3-5 mins that the patient stated were non-painful. Cervical examination was performed due to continued non-painful contractions, and her cervix was closed. She was transferred to labor and delivery for the remainder of her postoperative care.

The patient received betamethasone and magnesium postoperatively due to concern for possible preterm delivery due to initially non-reassuring fetal heart tones. With intrauterine resuscitation, delivery was not required and contractions spaced out. The patient was started on 5,000 units of heparin three times daily for VTE prophylaxis. 
Physical therapy was initiated while inpatient. She was discharged on postoperative day five with a 30-day supply of $30 \mathrm{mg}$ twice daily low-molecular-weight heparin (LMWH) after ambulating with a walker and had a spontaneous vaginal delivery at term.

Patient 2: A 34-year-old gravida 8 para 6 woman at 30 weeks of gestation presented to the emergency department with 3 weeks of severe hip pain after stepping off a curb. Plain film radiography demonstrated a minimally displaced right basicervical femoral neck fracture (Figure 1B). Her obstetric history included six preterm deliveries and an ectopic pregnancy treated surgically with a laparoscopic salpingectomy. She had inconsistently received intramuscular 17-hydroxyprogesterone injections during her pregnancy for prevention of preterm delivery. She had a past medical history of hypothyroidism, methadone dependence (185 mg daily since 21 weeks of gestation), and a 20-packyear smoking history. She was smoking $1 / 2$ pack of cigarettes per day throughout her pregnancy. Laboratory evaluation was remarkable for a low serum calcium $(8.1 \mathrm{mg} / \mathrm{dL})$ with a low serum albumin of $2.7 \mathrm{~g} / \mathrm{dL}$ and a low serum 25hydroxy vitamin $(7.1 \mathrm{ng} / \mathrm{mL})$.

The patient underwent an uncomplicated femoral neck pinning on the day of admission utilizing a $0.5 \%$ bupivacaine spinal anesthetic. Total preoperative and intraoperative radiation exposure was $9.6 \mathrm{mGy}$. Similar to the first case, a lead apron was used to shield the maternal abdomen during all images required pre-and- intraoperatively. Subcutaneous LMWH 30 mg twice daily was started postoperatively for VTE prophylaxis. She was transferred to labor and delivery for recovery and there were no postoperative complications. Both vitamin D and calcium were orally supplemented, physical therapy was initiated while inpatient, and antenatal fetal monitoring was conducted and was reassuring. The patient was ambulatory with the assistance of a walker and discharged to home on postoperative day 2. She subsequently presented to labor and delivery at 35 weeks and 5 days of gestation in preterm labor and had a vaginal delivery of a viable female fetus followed by an uneventful postpartum course.

Patient 3: A 24-year-old gravida 6 para 4 female at 31 weeks of gestation presented to the emergency department as a belted passenger in a 45-mph motor vehicle collision. Her pregnancy had been complicated by anemia and obesity. Plain film radiography demonstrated a mildly comminuted and displaced right femoral shaft fracture with foreshortening (Figure 1C). Her obstetric history included four previous full-term spontaneous vaginal deliveries and one elective abortion. She had no other significant past medical history. Laboratory evaluation was remarkable for a white blood cell count of $20.7 \times 10^{3} / \mu \mathrm{L}$ and hemoglobin of $9.1 \mathrm{~g} / \mathrm{dL}$, with a normal calcium level of $8.6 \mathrm{mg} / \mathrm{dL}$. She was transferred to labor and delivery where fetal monitoring was reassuring. Uterine tocometry showed contractions every 2-10 mins and the decision was made to give a $12 \mathrm{mg}$ dose of intramuscular betamethasone for fetal lung maturity prior to surgical intervention.

On hospital day 1 , the patient underwent an uncomplicated anterograde rod insertion of her right femur under general endotracheal anesthesia. Fetal monitoring before and during surgery was reassuring. Total preoperative and intraoperative radiation dosage was $135.2 \mathrm{mGy}$ and a lead apron was used to shield the maternal abdomen during all images. The patient was started on $30 \mathrm{mg}$ twice daily enoxaparin sodium for VTE prophylaxis, physical therapy as well as oral cholecalciferol and calcium acetate for daily supplementation. Throughout her postoperative course, the patient was not meeting ambulation goals necessary for discharge and on postoperative day 5 , she was diagnosed with preterm premature rupture of membranes. Azithromycin and amoxicillin were started as latency antibiotics. On postoperative day 7, the patient complained of increasing pain and pressure, the cervix was found to be fully dilated, and she vaginally delivered a live born male fetus at 32 weeks and 1-day gestation from the breech presentation. The patient had an unremarkable postpartum course and after ambulating with a walker was discharged home on postpartum day 2 .

\section{Discussion}

Orthopedic injury in pregnancy is an uncommon event, with most sources citing an incidence of $1-6 \%{ }^{3,4}$ Though rare, it is established that both the maternal and fetal morbidity and mortality are high, with some estimates of IUFD as high as $40.1 \%$ in patients with pelvic ring fractures. ${ }^{5}$ There have been case reports detailing the orthopedic operative management of acetabular and femoral fractures in pregnancy, but obstetric management before, during, and after these procedures is unclear. Literature on this topic is limited to one report of an acetabular fracture repaired in a viable pregnancy and a retrospective case series of 8 patients with gestational ages ranging from 5-26 weeks gestation at the time of surgery. ${ }^{6,7}$ Here we review obstetric peri-operative management of three cases of femoral fracture complicating second and third trimester pregnancies in a single university hospital, where a multi-disciplinary approach was implemented to 
mitigate the high morbidity associated with maternal femoral fractures in pregnancy.

The multidisciplinary approach in all three cases included the following teams: maternal-fetal medicine, orthopedics, anesthesiology, and neonatology After diagnosis of a long-bone fracture, maternal stabilization, and the need for operative intervention is decided, the appropriate anesthetic was determined. The patients we described were managed with different anesthetic techniques, two with a general anesthetic and one with neuraxial anesthesia. Review of literature revealed no robust data regarding which anesthetic route should be undertaken for non-obstetric surgery in a pregnant patient. Inhaled general anesthetic agents have not been found to be teratogenic at the levels given for surgery but have been associated with increased risk of preterm labor in the second trimester. ${ }^{8}$ In our case series, although all three surgeries were conducted at the same tertiary care center, the choice of anesthetic agent and route was individualized for each patient and planned surgical repair. The patient in case one had several injuries to the lower extremities, and the care of the patient in case three was complicated by maternal obesity, both of which may have made the conversion from neuraxial anesthesia to general during surgery challenging. Finally, the patient in case two was a good candidate for neuraxial anesthesia based on her body mass index and less complex fracture.

Intraoperative management is of critical importance in the management of the viable pregnancy with maternal femoral fracture. The obstetric team worked closely with the operating room personnel for proper positioning of the patients with left lateral tilt (approximately 15 degrees) to avoid compression of the inferior vena cava by the gravid uterus. The patients were offered continuous intraoperative fetal monitoring based on their desire for surgical intervention on fetal behalf after neonatology and maternalfetal-medicine preoperative consulation. ${ }^{8,9}$ Preoperative and intraoperative fetal protection with a lead apron placed over maternal abdomen was used to minimize fetal exposure to radiation. There is evidence that plates compared to intramedullary nailing confer decreased radiation doses. ${ }^{10}$ Intramedullary nailing was only used for femoral fracture repair in one of the included patients. Even though the radiation dose used in all three cases was minimal, this demonstrates the importance of multi-disciplinary preoperative planning to reduce the pre-and- intraoperative radiation exposure to the fetus.
Postoperatively, the patients received VTE prophylaxis with subcutaneous heparin or LMWH. ${ }^{11}$ The dosage recommended by the American College of Chest Physicians ${ }^{11}$ is $30 \mathrm{mg}$ enoxaparin sodium subcutaneous daily for 28-35 days. Modifications to this dosage and the length of therapy in the pregnant population with femur fracture is not known. Our patients had a planned 30-day regimen for their anticoagulation with $30 \mathrm{mg}$ BID of LMWH. In addition, intensive physical therapy was initiated on postoperative day 1 and was continued in the outpatient setting.

Data regarding the route of delivery in patients that have suffered a lower extremity fracture is lacking. One retrospective series reviewed pelvic fractures in women of childbearing age and found a cesarean section rate of $62 \%$ at a single institution. One case report of an acetabular femoral fracture that underwent fixation resulted in a vaginal delivery at term. ${ }^{6}$ In a retrospective case series with 8 patients that underwent late-preterm or term delivery, $50 \%$ had cesarean sections but their obstetric history was not reported. ${ }^{7}$ In our case series, all patients had spontaneous vaginal deliveries. The hypothesis for higher rates of cesarean deliveries in previous reports was suspected impaired ability of affected women to perform hip abduction. In our three cases, physical therapy was initiated in the immediate post-operative period with emphasis to continue working on hip abduction. All three patients were able to abduct their hips and place their legs in stirrups; therefore, cesarean section was reserved solely for obstetric indications.

The second case report we described is unique as the fracture occurred in a young woman in the absence of trauma. The etiology of that femur fracture was presumed to be osteoporotic in origin, secondary to smoking ${ }^{12}$ and methadone ${ }^{13}$ with low levels of calcium and vitamin D. Although the patient did not undergo bone mineral density testing, clinical diagnosis of osteoporosis was made in the presence of fragility fracture ${ }^{14}$ and she was started on vitamin D and calcium supplementation. Although some studies suggest that pregnancy itself is a risk factor for temporary osteoporosis, the data is overall inconclusive and rarely leads to clinical manifestations during pregnancy and postpartum. ${ }^{15-18}$ However, in the presence of additional risk factors, osteoporosis can complicate pregnancy, as occurred in our second case; this enhanced our awareness of the possibility of this diagnosis in a younger population.

In conclusion, a comprehensive multidisciplinary approach to successful management of femoral fracture in pregnancy was reviewed in this case report series. Despite different etiologies of femoral fracture, all women benefited from VTE prophylaxis with LMWH, early initiation of 
physical therapy with emphasis on hip abduction, supplementation with vitamin $\mathrm{D}$ and calcium, and all were able to safely achieve vaginal delivery.

\section{Disclosure}

The authors report no conflicts of interest in this work.

\section{References}

1. Almog G, Liebergall M, Tsafrir A, Barzilay Y, Mosheiff R. Management of pelvic fractures during pregnancy. Am J Orthop (Belle Mead NJ). 2007;36(11):E153-9.

2. Cannada LK, Barr J. Pelvic fractures in women of childbearing age. Clin Orthop Relat Res. 2010;468(7):1781-1789. doi:10.1007/s11999. 010-1333-5

3. Cannada LK, Pan P, Casey BM, McIntire DD, Shafi S, Leveno KJ. Pregnancy outcomes after orthopedic trauma. J Trauma. 2010;69 (3):694-8; discussion 698. doi:10.1097/TA.0b013e3181ec2b11

4. El Kady D, Gilbert WM, Xing G, Smith LH. Association of maternal fractures with adverse perinatal outcomes. Am J Obstet Gynecol. 2006;195(3):711-716. doi:10.1016/j.ajog.2006.06.067

5. Weinlein JC, Mashru RP, Perez EA, Johnson SE. Lateral compression-I pelvic ring injury: not benign to the developing fetus. J Orthop Trauma. 2018;32(2):100-103. doi:10.1097/BOT. 0000000000001030

6. Kloen P, Flik K, Helfet DL. Operative treatment of acetabular fracture during pregnancy: a case report. Arch Orthop Trauma Surg. 2005;125 (3):209-212. doi:10.1007/s00402-005-0798-2

7. Porter SE, Russell GV, Qin Z, Graves ML. Operative fixation of acetabular fractures in the pregnant patient. J Orthop Trauma. 2008;22(8):508-516. doi:10.1097/BOT.0b013e31816080d6

8. Ogle JA. Improving web site performance using commercially available analytical tools. Indian J Anaesth. 2010;468(10):2604-2611 doi:10.4103/0019-5049.179445
9. Nonobstetric surgery during pregnancy. Committee Opinion No. 696. American College of Obstetricians and Gynecologists. Obstet Gynecol. 2017;129:777-778. doi:10.1097/AOG.000000000 0002014

10. Labronici PJ, Lyra FS, Moreira IL, et al. Fractures of the distal extremity of the tibia treated with intramedullary nail or bridge plate: comparison of radiation exposure between the two methods. Rev Bras Ortop. 2010;45(2):132-135. doi:10.1016/S2255-4971(15) 30281-0

11. Falck-Ytter Y, Francis CW, Johanson NA, et al. Prevention of VTE in orthopedic surgery patients: antithrombotic Therapy and Prevention of Thrombosis, 9th ed: American College of Chest Physicians Evidence-Based Clinical Practice Guidelines. Chest. 2012;141 (2Suppl):e278S-e325S. doi:10.1378/chest.11-2404

12. Siris ES, Chen Y-T, Abbott TA, et al. Bone mineral density thresholds for pharmacological intervention to prevent fractures. Arch Intern Med. 2004;164(10):1108-1112. doi:10.1001/ archinte.164.10.1108

13. Teng $\mathrm{Z}, \mathrm{Zhu} \mathrm{Y}, \mathrm{Wu} \mathrm{F}$, et al. Opioids contribute to fracture risk: a meta-analysis of 8 cohort studies. PLoS One. 2015;10(6):e0128232. doi:10.1371/journal.pone.0128232

14. Siris ES, Adler R, Bilezikian J, et al. The clinical diagnosis of osteoporosis: a position statement from the National Bone Health Alliance Working Group. Osteoporos Int. 2014;25(5):1439-1443. doi:10.1007/s00198-014-2655-z

15. Karlsson MK, Ahlborg HG, Karlsson C. Maternity and bone mineral density. Acta Orthop. 2005;76(1):2-13. doi:10.1080/ 00016470510030274

16. Naylor KE, Iqbal P, Fledelius C, Fraser RB, Eastell R. The effect of pregnancy on bone density and bone turnover. J Bone Miner Res. 2000;15(1):129-137. doi:10.1359/jbmr.2000.15.9.1798

17. Aj B, Topping J, Durham B, Rg F, Wd F. A detailed assessment of alterations in bone turnover, calcium homeostasis, and bone density in normal pregnancy. $J$ Bone Miner Res. 2000;15(3):557-563. doi:10.1359/jbmr.2000.15.9.1798

18. Sowers M, Crutchfield M, Jannausch M, Updike S, Corton G. A prospective evaluation of bone mineral change in pregnancy. Obstet Gynecol. 1991;77(6):841-845.
International Journal of Women's Health

\section{Publish your work in this journal}

The International Journal of Women's Health is an international, peerreviewed open-access journal publishing original research, reports, editorials, reviews and commentaries on all aspects of women's healthcare including gynecology, obstetrics, and breast cancer. The manuscript management system is completely online and includes a very quick and fair peer-review system, which is all easy to use. Visit http://www.dovepress.com/testimonials.php to read real quotes from published authors. 